\title{
LITERATUR
}

\section{Der Vertrag von Lissabon und die ,neue 6 Europäische Union aus der rechtswissenschaftlichen Perspektive}

\author{
Marcus Hornung*
}

Als am 29. Mai und 1. Juli 2005 die Bevölkerungen Frankreichs und der Niederlande in Referenden gegen die Ratifikation des wenige Monate zuvor unterzeichneten Vertrags über eine Verfassung für Europa stimmten, bedeutete dies das vorläufige Scheitern eines rund fünf Jahre andauernden Verfassungsprojekts. Die Staats- und Regierungschefs entschieden sich daher für den Rückzug in eine rund zweijährige „Reflexionsphase“, an deren Ende der Auftrag stand, den ehemaligen Verfassungsprozess nunmehr als „Reformprozess“ fortzusetzen. ${ }^{1}$ Bereits auf seiner Sitzung vom 21./22. Juni 2007 erteilte der Europäische Rat einer neuerlichen Regierungskonferenz das Mandat, einen Reformvertrag ,zur Änderung der bestehenden Verträge auszuarbeiten [...]. Das Verfassungskonzept, das darin bestand, alle bestehenden Verträge aufzuheben und durch einen einheitlichen Text mit der Bezeichnung, Verfassung' zu ersetzen, wird aufgegeben.“2 Am 13. Dezember 2007 wurde der Vertrag von Lissabon ${ }^{3}$ von den Staats- und Regierungschefs unterzeichnet - vom Glanz des ambitionierten Verfassungsprojekts blieb schließlich ein gewöhnlicher Reformvertrag.
Rudolf Streinz/Christoph Ohler/Christoph Herrmann: Der Vertrag von Lissabon zur Reform der EU. Einführung mit Synopse, 3. Aufl., C.H. Beck Verlag: München 2010, ISBN 978-3-406-59776-3; 430 Seiten, $42,00 €$.

Jean-Claude Piris: The Lisbon Treaty. A Legal and Political Analysis, Cambridge University Press: Cambridge 2010, ISBN 978-0-521-14234-2; 426 Seiten, 36,36 €.

Hermann-Josef Blanke/Stelio Mangiameli (Hrsg.): The European Union after Lisbon. Constitutional Basis, Economic Order and External Action, Springer-Verlag: Berlin/Heidelberg 2012, ISBN 978-3-642-19506-8; 582 Seiten, 106,95 €.

Dabei übernahm der neue Vertrag von Lissabon große Teile der materiellen Substanz des Verfassungsvertrags. Trotz der Aufgabe von Staatlichkeit suggerierenden Termini wie ,Verfassung', europäisches ,Gesetz', ,Außenminister' oder Symboliken wie der Flagge und Hymne, verblieben wesentliche Neuerungen inhaltlich unverändert im Vertragstext: Die Verleihung ausdrücklicher Rechtspersönlichkeit an die Union, ${ }^{4}$ die Überwindung des Maastrichter ,Drei-Säulen-Modells‘ ${ }^{5}$ die Einfüh-

\footnotetext{
* Marcus Hornung, M.E.S., Wissenschaftlicher Mitarbeiter, Jean-Monnet-Professur für Europäische Integration, Technische Universität Chemnitz.

1 Vgl. Rat der Europäischen Union: Europäischer Rat (Brüssel) 15./16. Juni 2006. Schlussfolgerungen des Vorsitzes, 10633/1/06, S. 16-17.

2 Rat der Europäischen Union: Europäischer Rat (Brüssel) 21./22. Juni 2007. Schlussfolgerungen des Vorsitzes, $11177 / 1 / 07$, S. 15.

3 Vertrag von Lissabon zur Änderung des Vertrags über die Europäische Union und des Vertrags zur Gründung der Europäischen Gemeinschaft, unterzeichnet in Lissabon am 13. Dezember 2007, in: Amtsblatt der EU, Nr. C 306 vom 17. Dezember 2007, S. 1-271.

4 Vgl. Art. 47 Vertrag über die Europäische Union (EUV).

5 Die Aufgabe des ,Drei-Säulen-Modells‘ ergibt sich aus der Überführung sämtlicher Politikbereiche in eine einheitliche Europäische Union mit Rechtspersönlichkeit. Vgl. Art. 47 EUV.
} 
rung der , doppelten Mehrheit ${ }^{6}$ und die Schaffung eines permanenten Präsidenten ${ }^{7}$ des Europäischen Rates sind nur wenige Beispiele unter vielen. Möglich ist, dass die inhaltliche Nähe des neuen Vertrags von Lissabon zum gescheiterten Verfassungsvertrag Ursache des erneut stockenden Ratifikationsprozesses war. Trotz allem konnte der Vertrag von Lissabon am 1. Dezember 2009 in Kraft und damit eine ,neue‘ Europäische Union die Rechtsnachfolge der Europäischen Gemeinschaft antreten. ${ }^{8}$

Spätestens seit seiner Unterzeichnung wurde der Vertrag von Lissabon umfangreich durch die Rechts- sowie Politikwissenschaft aufgearbeitet, bietet doch nicht nur seine Entstehungsgeschichte, sondern auch sein Reformgehalt unzählige Anknüpfungspunkte für vertiefte Analysen. Aktuelle Publikationen zum Vertrag von Lissabon sind denn auch häufig durch diese Dualität gekennzeichnet: Sie haben zwar den unfreiwilligen Übergang vom gescheiterten Verfassungs- zum Reformvertrag zur Grundlage, aber fokussieren sodann fast immer auch auf die Gestalt der neuen Lissabon-Union im Sinne einer Erfassung des konsolidierten Bestandes. Dieser Bestandsaufnahme folgt häufig die Betrachtung ausgewählter durch den Vertrag von Lissabon reformierter Politikfelder.

Nach diesem Muster sind auch die drei hier als Beispiele der Flut an rechtswissenschaftlichen Publikationen besprochenen Werke aufgebaut, deren Charaktere sich ansonsten erheblich unterscheiden: Mit „Der Vertrag von Lissabon zur Reform der EU“9 legten die Rechtswissenschaftler Rudolf Streinz, Christoph Ohler und Christoph Herrmann schon 2010 eine deutsch- sprachige Einführung mit Synopse vor, die eine thematische Näherung und Vertiefung bietet. Im gleichen Jahr erschien die englischsprachige Monografie „The Lisbon Treaty. A Legal and Political Analysis " ${ }^{\text {10 }}$ von Jean-Claude Piris, dem Generaldirektor des Juristischen Dienstes des Rates. Piris eröffnet dem Leser praktische Einblicke, fundierte Bewertungen und kritische Sichtweisen. Der Sammelband „The European Union after Lisbon"11 von Hermann-Josef Blanke und Stelia Mangiameli zählt hingegen zu den einschlägigen Neuerscheinungen im Themenfeld. Er bietet neben Analyse auch politikfeldspezifische Expertise und Diskussionsansätze.

\section{Streinz, Ohler, Herrmann: Näherung, Einführung und Vertiefung}

Vor dem Hintergrund der Entstehungsgeschichte des Vertrags von Lissabon verwundert es kaum, dass der Band von Streinz, Ohler und Herrmann ganz im Zeichen des gescheiterten Verfassungsvertrags steht, erschien doch die erste Auflage des vorliegenden Buchs noch unter dem Titel „Die neue Verfassung für Europa". ${ }^{12}$ So wurde auch der bewährte Aufbau - im Kern bestehend aus einer Herleitung der Verfassungsidee im europäischen Kontext, einer europarechtlichen Einführung sowie einer angehangenen Textsynopse - für die aktuell vorliegende dritte Auflage übernommen und um die zum Zeitpunkt ihres Erscheinens jüngste Rechtsprechung wie der Lissabon-Entscheidung des Bundesverfassungsgerichtes ${ }^{13}$ ergänzt und aktualisiert. Mit einem in den Vertrag von Lissabon einführenden Band wendet sich das Autorentrio insbesondere an Studierende der Rechtswissenschaft im Haupt- und Nebenfach und ausdrücklich auch an europa-

6 Vgl. Art. 16 Abs. 4 EUV.

7 Vgl. Art. 15 Abs. 5 EUV.

8 Vgl. Art. 1 Abs. 3 S. 3 EUV.

9 Rudolf Streinz/Christoph Ohler/Christoph Herrmann: Der Vertrag von Lissabon zur Reform der EU. Einführung mit Synopse, 3. Aufl., München 2010.

10 Jean-Claude Piris: The Lisbon Treaty. A Legal and Political Analysis, Cambridge 2010.

11 Hermann-Josef Blanke/Stelio Mangiameli (Hrsg.): The European Union after Lisbon. Constitutional Basis, Economic Order and External Action, Berlin/Heidelberg 2012.

12 Rudolf Streinz/Christoph Ohler/Christoph Herrmann: Die neue Verfassung für Europa. Einführung und Synopse, 1. Aufl., München 2005.

13 BVerfG, 2 BvE 2/08 vom 30.6.2009. 
rechtlich interessierte Praktiker sowie Fachfremde.

Um diesem Anspruch gerecht zu werden, kann auf die Vermittlung von Basiswissen nicht verzichtet werden: Die Autoren führen den Leser mithilfe einer ausgedehnten Einleitung in die Verfassungsidee im europäischen Kontext an die Entstehungsgeschichte und schließlich die Konzeption des Vertrags von Lissabon heran. Diese Einführung erinnert deutlich an die Verfassungstradition, in der sowohl die dritte Auflage des Buchs als auch der Vertrag von Lissabon selbst stehen. Bearbeiter Streinz führt den Leser tiefer in die Thematik ein, als man es von einem Einführungswerk erwarten würde: Die Gründlichkeit etwa, in der der Verfassungsgedanke bis ins 18. Jahrhundert zurückverfolgt und mit der in der Folge seine Anwendbarkeit auf die europäische Ebene diskutiert wird, ${ }^{14}$ übertrifft jene einschlägiger Europarechtslehrbücher. Von Detailtiefe zeugt auch, dass nach einer knappen Übersicht über die auf die Unterzeichnung des Vertrags von Lissabon folgenden Ratifikationen in den 27 Mitgliedstaaten das deutsche Beispiel gesondert behandelt wird. Der neue Abschnitt zum Lissabon-Urteil des Bundesverfassungsgerichts ${ }^{15}$ fasst - auch für Nicht-Juristen problemlos nachvollziehbar - alle relevanten Diskussionsbestandteile zusammen.

Die zwei folgenden Teile ,Grundlagen der Europäischen Union“16 und „Die Rechtsordnung der Union"17 liefern in der Qualität eines Lehrbuchs die angesprochene Bestandserfassung der Union ,nach Lissabon“. Lesern ohne europarechtliche Vorbildung bieten diese Kapitel etwa zu den Organen ${ }^{18}$ oder der Kompetenz- ordnung $^{19}$ - einen vollwertigen Einstieg in die rechtlichen Grundlagen der Europäischen Union. Leser mit entsprechender Vorbildung dürften hingegen davon profitieren, dass in allen Abschnitten gezielt die Änderungen durch den Vertrag von Lissabon fokussiert und dem alten Rechtsstand gegenübergestellt werden, wie etwa die Bildung der neuen, doppelten Mehrheit $^{620}$ oder die Neuformulierung der Flexibilitätsklausel in Art. 352 Vertrag über die Arbeitsweise der Europäischen Union (AEUV). ${ }^{21}$

Diesen Spagat versuchen die Autoren auch in den folgenden Teilen zum auswärtigen Handeln der Union ${ }^{22}$ und zum Raum der Freiheit, der Sicherheit und des Rechts ${ }^{23}$ (RFSR). Stärker als zuvor wird hier jedoch exklusiv auf die Neuerungen des Vertrags von Lissabon abgestellt, was am Beispiel des auswärtigen Handelns schon durch die Voranstellung der Verhandlungsgeschichte des Politikfeldes bis hin zum Vertrag von Lissabon ${ }^{24}$ verdeutlicht wird. Der fünfte Teil zum RFSR schließlich kann schon aufgrund seines nur knappen Umfanges kaum noch als eigenständige Einführung gelesen werden.

Das von Streinz, Ohler und Herrmann vorgelegte Werk macht sich um eine ausnahmslos fundierte und trotzdem knappe Einführung in den Reformgehalt des Vertrags von Lissabon verdient. Dass diese Einführung auch fortgeschrittenen Lesern ein Einstieg zu vertiefter Analyse sein kann, wird nicht zuletzt durch unzählige kapitelbezogene Literaturhinweise sowie die beigefügte Textsynopse bewiesen. Es gelingt den Autoren somit, dem selbst gesetzten Anspruch des Buchs, einen Beitrag für

14 Streinz/Ohler/Herrmann: Der Vertrag von Lissabon zur Reform der EU, 2010, S. 1-16.

15 Ebenda, S. 27-36.

16 Ebenda, S. 45-90.

17 Ebenda, S. 91-128.

18 Ebenda, S. 57-72.

19 Ebenda, S. 103-111.

20 Ebenda, S. 66-67.

21 Ebenda, S. 110-111.

22 Ebenda, S. 129-156.

23 Ebenda, S. 157-166.

24 Ebenda, S. 129-131. 
ein besseres Verständnis des europäischen Integrationsprozesses $\mathrm{zu}$ leisten, ${ }^{25}$ sogar zielgruppenübergreifend gerecht zu werden.

\section{Piris: Praxis, Bewertung und Kritik}

Aus den vielen englischsprachigen Monografien sticht jene von Piris heraus, handelt es sich dabei doch um das Werk eines ausgewiesenen Europa-Praktikers, welches noch dazu von einem Vorwort Angela Merkels geschmückt wird. „The Lisbon Treaty. A Legal and Political Analysis“ folgt auf das 2006 erschienene „The Constitution for Europe: A Legal Analysis“ ${ }^{26}$ Die Gegenüberstellung dieser zwei Titel zeugt von einem Paradigmenwechsel in Piris' Werken: Tatsächlich nimmt sich der Verfasser vor, die Neuerungen des Vertrags von Lissabon im Kontext und nicht nur rechtlich zu beschreiben: ,to present the main elements of the Lisbon Treaty and to explain their legal and political meaning and effect, putting them in their historical and political context. “27 Das Inhaltsverzeichnis zeugt wiederum von der bewährten Herangehensweise vieler Analysen des Vertrags von Lissabon: Auf ein Kapitel zur Entstehungsgeschichte des Reformvertrags folgen insgesamt sieben Abschnitte, die seine Veränderungen an der Union selbst und eine Auswahl ihrer Politikfelder behandeln. Ein umfangreicher Anhang diskutiert schließlich noch die Lissabon-Urteile des tschechischen und des deutschen Verfassungsgerichts und bündelt tabellarische Übersichten. Während das Werk Piris' damit konzeptionelle Parallelen zum Werk von Streinz, Ohler und Herrmann aufweist, ist die methodische Vorgehensweise unterschiedlich: Piris arbeitet konsequent die Reformen des Vertrags von Lissabon ab, während Streinz, Ohler und Herrmann eher die Union ,nach Lissabon “ unter exklusiver Berücksichtigung der Reformen beschrei- ben. Die Lektüre von „The Lisbon Treaty. A Legal and Political Analysis" erfordert daher zumindest gewisse Vorkenntnisse der Unionsstruktur und -funktionsweise.

Das einleitende Kapitel „The origins and birth of the Lisbon Treaty" 28 steht im Zeichen des gescheiterten Verfassungsprozesses: Anschaulich schildert Piris nicht nur Tagungen des Europäischen Rates, Regierungskonferenzen, Ratifikationsprozesse und Referenden, sondern auch sämtliche politische Unstimmigkeiten, Winkelzüge und Lagerbildungen, die seit der Erklärung von Laeken zur Zukunft der Europäischen Union ${ }^{29}$ die Primärrechtsreform begleitet haben. Der Autor gestaltet diesen Abschnitt so praxisnah wie möglich und macht den Lesern namentlich Positionen politischer Akteure und ihr jeweiliges Verhandlungsgewicht transparent. Das tiefgründige Wissen um diese politischen Prozesse schöpft Piris aus seiner Tätigkeit als Generaldirektor des Juristischen Dienstes des Rates, in deren Rahmen er an der Ausarbeitung des Verfassungsvertrags sowie des Vertrags von Lissabon beteiligt war. Besonderes Verdienst dieses Abschnittes ist, dass Piris es immer wieder - zum Beispiel durch den Einbezug zahlreicher Primärtexte schafft, Recht und Politik nachvollziehbar miteinander zu verknüpfen. Dass der Verfasser seine eingängigen Erläuterungen zum Lissabon-Urteil des Bundesverfassungsgerichts nicht direkt in seinen Text einbindet, sondern in einen Anhang ${ }^{30}$ verschiebt, ist hingegen schade.

Die folgenden Kapitel führen den Leser, nach Sachbereichen untergliedert, durch die wesentlichen Reformen des Vertrags von Lissabon: In „General provisions" ${ }^{\text {"31 }}$ erläutert Piris zum Beispiel die neu aufgenommenen Werte, die Neuordnung der Kompetenzkategorien und die

25 Vgl. ebenda, Vorwort zur 3. Auflage.

26 Jean-Claude Piris: The Constitution for Europe: A Legal Analysis, Cambridge 2006.

27 Piris: The Lisbon Treaty, 2010, S. 6.

28 Ebenda, S. 7-70.

29 Erklärung von Laeken zur Zukunft der Europäischen Union, in: Rat der Europäischen Union: Schlussfolgerungen des Vorsitzes. Europäischer Rat (Laeken) 14. und 15. Dezember 2001, SN 300/1/01, Anlage I, S. 19-26.

30 Piris: The Lisbon Treaty, 2010, S. 341-358.

31 Ebenda, S. 71-111. 
Rechtspersönlichkeit der Union. Dem oben zitierten Anspruch seines Werkes wird der Verfasser dadurch gerecht, dass er politische Diskurse im Hintergrund einer Bestimmung aufnimmt - etwa zur Darstellung der Kompetenzabgrenzung $^{32}$ - oder durch den Einbezug einschlägiger Fälle - etwa Costa/ENEL im Fall des Vorranges ${ }^{33}$ - historische Bezüge aufzeigt. Ähnlich stellen sich die Kapitel zum Grundrechtsschutz ${ }^{34}$ sowie dem institutionellen Gefüge ${ }^{35}$ dar. Piris leitet aus seiner juristischen Betrachtung immer wieder auch politische Fragen $^{36}$ ab. Dies geht so weit, dass der Verfasser im Abschnitt „Democracy“37 eine fundierte Diskussion des vermeintlichen Demokratiedefizits der Union entwickelt.

Wie schon Streinz, Ohler und Herrmann unterzieht auch Piris schließlich die Reform ausgewählter Politikbereiche einer vertieften Analyse: Justiz und Inneres, ${ }^{38}$ auswärtige Angelegenheiten $^{39}$ sowie Finanzen, Wirtschaft und Soziales, die der Autor in einem gemeinsamen Kapitel mit anderen internen Politiken behandelt. ${ }^{40}$ Die Zusammenfassung von Landwirtschaft, Fischerei, Energie, Raumfahrt und Sport auf nur wenigen Seiten kann der Bedeutung dieser Politikbereiche kaum gerecht werden. In die Reformen im Bereich Justiz und Inneres sowie Auswärtiges wiederum leitet Piris zugunsten der besseren Verständlichkeit jeweils mit einer nützlichen Darstellung ${ }^{41}$ des Rechtsstandes vor Inkrafttreten des Vertrags von Lissabon ein.

Besondere Erwähnung verdient Piris' Schlussteil, ${ }^{42}$ der weit über eine Zusammenfassung der
Lissaboner Primärrechtsreformen hinausgeht. Ausgehend von seiner Analyse wirft der Verfasser abermals offene Fragen auf, in deren Diskussion er deutlich Stellung bezieht: Man merkt dem Praktiker Piris an, dass er der Symbolkraft eines kohärenten Verfassungsvertrags nachtrauert: ,while that Treaty was an attempt to mark a historic step in a federalist direction, this ambition has been abandoned with the Lisbon treaty. "43 Nüchtern hingegen hinterfragt der Autor dann die Reichweite der LissabonReformen und moniert, dass Asymmetrien in der Architektur der Union nicht beseitigt wurden und noch immer Legitimationsdefizite bestehen. Mit Blick auf das gesamte Werk fällt dann auch sein Fazit erstaunlich kritisch aus, indem er einräumt, dass der Vertrag von Lissabon trotz der Übernahme der materiellen Substanz des Verfassungsvertrags, „does not represent a great leap forward." 44

Insgesamt legt Piris mit, ,The Lisbon Treaty. A Legal and Political Analysis" eine gut lesbare Übersicht über die Reformen des Vertrags vor, die vor allem durch ihre Praxisnähe beeindruckt: Die Monografie bietet fortgeschrittenen Lesern nicht nur eine fundierte Beschreibung der wesentlichen Reformbestandteile, sondern auch politische Einblicke, kritische Fragen und ambitionierte Denkanstöße. Einige dieser offenen Fragen greift Piris in seinem jüngst erschienenen Werk „The Future of Europe: Towards a Two-Speed EU?“45 selbst auf.

\section{Blanke, Mangiameli: Analyse, Expertise und Diskussion}

Der von Blanke und Mangiameli herausgegebene Sammelband „The European Union after

32 Ebenda, S. 76-78.

33 Ebenda, S. 79-82.

34 Ebenda, S. 146-166.

35 Ebenda, S. 204-237.

36 Ebenda, S. 145, 237.

37 Ebenda, S. 112-145.

38 Ebenda. S. 167-203.

39 Ebenda, S. 238-287.

40 Ebenda, S. 288-323.

41 Ebenda, S. 167-177, 238-243.

42 Ebenda, S. 324-340.

43 Ebenda, S. 327.

44 Ebenda, S. 339.

45 Jean-Claude Piris: The Future of Europe: Towards a Two-Speed EU?, Cambridge 2012. 
Lisbon. Constitutional Basis, Economic Order and External Action" folgt auf einen Vorgänger der gleichen Herausgeber, der noch 2006 den Verfassungsvertrag behandelt hatte. ${ }^{46}$ Der nun erschienene Sammelband bündelt in 24 Aufsätzen unter anderem Ergebnisse zweier Konferenzen in Erfurt (2008) und Rom (2010) von namhaften Autoren aus acht Nationen. Der Ansatz dieses Sammelbandes unterscheidet sich grundsätzlich von jenem der bereits rezensierten Werke: Blanke und Mangiameli werfen gemeinsam mit ihren Autoren Schlaglichter auf die ,neue' Union und analysieren damit nicht den Reformvertrag selbst, sondern vorrangig die Gestalt der durch ihn reformierten Union. Dementsprechend richtet sich das Werk eher an das Fachpublikum mit europarechtlichen Vorkenntnissen denn an Einsteiger. Diesen hätte etwa eine Einführung ${ }^{47}$ den fachlichen Zugang erleichtert. Wie der Titel andeutet, entscheiden sich die Herausgeber ebenfalls für die Analyse zweier Fallbeispiele für durch den Reformvertrag veränderte Politikbereiche-Gemeinsame Außen- und Sicherheitspolitik (GASP) sowie Wirtschafts- und Währungsunion -, die auf eine Bestandserfassung der „Constitutional Basis“ folgt.

Dieser erste Teil ${ }^{48}$ hat auch bei Blanke und Mangiameli den steinigen Weg vom Verfassungsvertrag zum Vertrag von Lissabon zur Grundlage: In seinem einleitenden Beitrag ${ }^{49}$ hinterfragt Antonio D'Atena, ob das Scheitern des Verfassungsvertrags auch die Abkehr von der Idee einer europäischen Verfassung insgesamt nach sich ziehen müsse. Sodann analysieren weitere Autoren jene Wesenszüge der Union, die durch den Vertrag von Lissabon angetastet wurden - etwa ihre nunmehr eindeutige Rechtspersönlichkeit, ${ }^{50}$ die Einbindung der Charta der Grundrechte, ${ }^{51}$ oder die ausdrückliche Option des Austritts eines Mitgliedstaats. ${ }^{52}$ Die Aufsätze sind von hoher individueller Qualität und durchdringen die reformierten Bereiche deutlich tiefer, als das in einer der vorgestellten Einführungen möglich gewesen wäre. Ausgesprochen lesenswert ist unter anderem der Überblicksbeitrag des KoHerausgebers Mangiameli zu „The Institutional Design of the European Union After Lisbon" ${ }^{53}$ Statt - wie viele andere Autoren - die Organe und Institutionen einzeln auf die Veränderungen durch den Vertrag von Lissabon hin zu prüfen, wählt der Autor einen alternativen Zugang: Mangiameli geht von Art. 10 Abs. 1 EUV aus, der besagt, dass die Arbeitsweise der Union auf der repräsentativen Demokratie beruht. In der Folge werden die Organe und Institutionen der Lissabon-Union jeweils auf ihre Fähigkeit hin analysiert, diesem Anspruch Rechnung zu tragen.

Der zweite Teil des Buchs zur Wirtschafts- und Finanzverfassung der Union ${ }^{54}$ ist - bezogen auf den Umfang des Sammelbandes - knapp gehalten und überzeugt durch seinen stringent deduktiven Aufbau: In einem Überblicksarti-

46 Hermann-Josef Blanke/Stelio Mangiameli: Governing Europe under a Constitution: The Hard Road from the European Treaties to a European Constitutional Treaty, Heidelberg 2006.

47 Ein gutes Beispiel ist etwa: Hermann-Josef Blanke/Stelio Mangiameli: Introduction, in: Blanke/Mangiameli: Governing Europe under a Constitution, 2006, S. XXV-LXIV.

48 Blanke/Mangiameli: The European Union after Lisbon, 2012, S. 3-365.

49 Antonio D'Atena: The European Constitution's Prospects, in: Blanke/Mangiameli: The European Union after Lisbon, 2012, S. 3-19.

50 Daniel Thürer/Pierre-Yves Marro: The Union's Legal Personality: Ideas and Questions Lying Behind the Concept, in: Blanke/Mangiameli: The European Union after Lisbon, 2012, S. 47-70.

51 Eduardo Gianfrancesco: The Charter of Fundamental Rights of the Union as a Source of Law, in: Blanke/ Mangiameli: The European Union after Lisbon, 2012, S. 295-310. Auch: Herrmann-Josef Blanke: The Protection of Fundamental Rights in Europe, in: Blanke/Mangiameli: The European Union after Lisbon, 2012, S. $159-232$.

52 Anna Wyrozumska: Withdrawal from the Union, in: Blanke/Mangiameli: The European Union after Lisbon, 2012, S. 343-365.

53 Stelio Mangiameli: The Institutional Design of the European Union After Lisbon, in: Blanke/Mangiameli: The European Union after Lisbon, 2012, S. 93-128.

54 Blanke/Mangiameli: The European Union after Lisbon, 2012, S. 368-419. 
kel leitet Blanke $e^{55}$ die Wirtschaftsverfassung der Union her. Dieser Aufsatz ist umfassend beginnend mit der Entwicklung des Binnenmarktes und endend mit einem Ausblick auf die Zukunft der Wirtschafts- und Währungsunion - und holt damit den Leser beim individuellen Wissensstand ab. Auch legt er ein solides Fundament für die Lektüre des Kapitels von Ulrich Häde,${ }^{56}$ der die Lissabon-Reformen der Wirtschafts- und Währungsunion im Detail behandelt, ohne vorab Grundlagen wiederholen zu müssen. Häde fokussiert insbesondere die Bedenken gegen die durch den Vertrag von Lissabon verliehene Organstellung der Europäischen Zentralbank. Die Herausgeber entscheiden sich zum Abschluss des zweiten Teils, die Wettbewerbspolitik als Teilbereich der Wirtschaftsverfassung vertieft zu behandeln: Paul Adriaanse ${ }^{57}$ untersucht in seinem Beitrag die praktische Durchsetzung des Beihilferechts - ein Thema, das vor allem durch die Wirtschafts- und Finanzkrise erheblich an Relevanz gewonnen hat.

Der dritte und letzte Teil zur bereits intensiver erforschten GASP ${ }^{58}$ kommt ohne ein Grundlagenkapitel aus und behandelt ohne Umschweife Innovationen wie den ,Doppelhut ${ }^{\text {' des Ho- }}$ hen Vertreters der Union für Außen- und Sicherheitspolitik $^{59}$ oder die Ständige Strukturierte Zusammenarbeit im Rahmen der Gemeinsamen Sicherheits- und Verteidigungspolitik. ${ }^{60}$ Ausgehend vom Wissen über diese politikfeldspezifischen Neuerungen werden sie in der Folge durchaus konträr diskutiert, etwa wenn Ramses A. Wessel ${ }^{61}$ der These nachgeht, die GASP habe mit dem Vertrag von Lissabon einen Teil ihres intergouvernementalen Charakters verloren und Daniel Thym ${ }^{62}$ dieser These durch eine Analyse der Rolle des Politischen und Sicherheitspolitischen Komitees entgegentritt. Hierdurch gewinnt dieser dritte Abschnitt an Spannung und lädt zur eigenen vertieften Auseinandersetzung ein. Für das Verständnis und die Bewertung der GASP besonders hilfreich ist der Aufsatz Günter Sautters zu ihrer Finanzierung: ${ }^{63}$ Zum komplexen Finanzierungssystem existieren derzeit kaum vergleichbar konkrete und aktuelle Beiträge.

Das Verdienst des von Blanke und Mangiameli vorgelegten Sammelbandes ist daher neben der Konsolidierung der rechtswissenschaftlichen Forschung zum Vertrag von Lissabon auch die Anwendung neuer Analyseansätze. Es gelingt den Herausgebern damit, informierten Lesern eine anspruchsvolle und aktuelle Folgelektüre - etwa zu den bereits besprochenen Werken anzubieten.

Wenn einleitend festgestellt wurde, dass auf den gescheiterten Verfassungsvertrag mit dem Vertrag von Lissabon ein gewöhnlicher Reformvertrag folgte, so bezog sich dieser suggerierte Makel allein auf den Innovationsmodus, nicht aber auf die beinhalteten Reformen. Tatsächlich bildet der Vertrag von Lissabon eine Zäsur, da er sowohl die institutionelle Struktur der Union als auch ihre Politiken maß-

55 Herrmann-Josef Blanke: The Economic Constitution of the European Union, in: Blanke/Mangiameli: The European Union after Lisbon, 2012, S. 369-420.

56 Ulrich Häde: The Treaty of Lisbon and the Economic and Monetary Union, in: Blanke/Mangiameli: The European Union after Lisbon, 2012, S. 421-442.

57 Paul Adriaanse: Public and Private Enforcement of EU State Aid Law. Legal Issues of Dual Vigilance by the Commission and National Courts, in: Blanke/Mangiameli: The European Union after Lisbon, 2012, S. 443-467.

58 Blanke/Mangiameli: The European Union after Lisbon, 2012, S. 469-582.

59 Eileen Denza: The Role of the High Representative of the Union for Foreign Affairs and Security Policy, in: Blanke/Mangiameli: The European Union after Lisbon, 2012, S. 481-494.

60 Sebastian Graf von Kielmansegg: Permanent Structured Cooperation: A new Mechanism of Flexibility, in: Blanke/Mangiameli: The European Union after Lisbon, 2012, S. 551-566.

61 Ramses A. Wessel: Initiative and Voting in Common Foreign and Security Policy: The New Lisbon Rules in Historical Perspective, in: Blanke/Mangiameli: The European Union after Lisbon, 2012, S. 495-516.

62 Daniel Thym: The Intergovernmental Branch of the EU's Foreign Affairs Executive, in: Blanke/Mangiameli: The European Union after Lisbon, 2012, S. 517-532.

63 Günter Sautter: The Financing of Common Foreign and Security Policy - on Continuity and Change, in: Blanke/ Mangiameli: The European Union after Lisbon, 2012, S. 567-582. 
geblich verändert hat. Aus diesen Umständen erwuchs gesteigerter Publikationsbedarf: Interessierte Bürger, Studenten und Wissenschaftler vieler Disziplinen mussten ad hoc mit europarechtlichen Informationen versorgt werden. Entsprechend differenzierten Ansprüchen muss auch die Literaturlandschaft rund um den Reformvertrag genügen. Während alle drei hier ausgewählten Werke unbedingt empfehlenswert sind, zeichnen sie sich durch individuelle Stärken aus - Streinz, Ohler und Herrmann durch die gelungene Einheit aus Einführung und Gründlichkeit, Piris durch den eingängigen Praxisbezug sowie Blanke und Mangiameli durch die rechtswissenschaftliche Detailtiefe.

\section{Jahrbuch \\ Extremismus \& Demokratie}

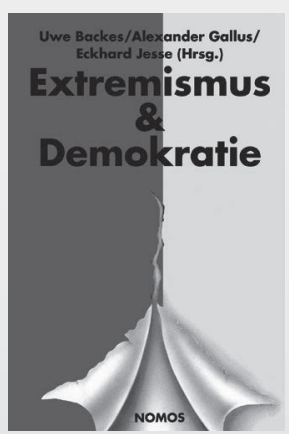

Band 24 des Jahrbuches gliedert sich wie jedes Jahrbuch zuvor in die bewährten drei Rubriken „Analysen“, „Daten, Dokumente, Dossiers“ und „Literatur". Die Entwicklung im politi-
Jahrbuch Extremismus \& Demokratie (E \& D)

24. Jahrgang 2012

Herausgegeben von Uwe Backes, Alexander Gallus und Eckhard Jesse 2012, 534 S., geb., 54,-€ ISBN 978-3-8329-7999-7

schen Extremismus wird nachgezeichnet - vor allem im Jahr 2011 und vor allem in Deutschland, aber auch darüber hinaus.
Weitere Informationen: www.nomos-shop.de/20002 